\title{
Der Future Skills Turn
}

William Ross Ashby wurde am 6. September 1903 in London in England geboren und starb am 15. November 1972. Er war ein britischer Psychiater und Pionier in der Kybernetik, dem Studium komplexer Systeme und gilt als eine der einflussreichsten Personen in den Systemwissenschaften (Klir 1978). Seine Werke Einführung in die Kybernetik (1974) und Design for a Brain (1952) waren seit ihrem Erscheinen in den 1950er Jahren einflussreich in den Wissenschaften von komplexen Systemen, damals unter dem Schlagwort Kybernetik bekannt. Obwohl er in der Wissenschaft komplexer Systeme so bedeutend war, ist er heute dennoch weitaus weniger bekannt als etwa Norbert Wiener oder Herbert A. Simon. Ashbys Gesetz trägt seinen Namen und lieferte die wissenschaftliche Grundlage für das homöostatische Prinzip und die Prinzipien der Selbstorganisation. Das Gesetz von der erforderlichen Varietät (in Englisch Law of Requisite Variety) gehört zu den zentralen Erkenntnissen der Kybernetik (Ashby 1974).

Das Gesetz besagt, dass ein System, welches ein anderes steuert, umso mehr Störungen in dem Steuerungsprozess ausgleichen kann, je größer seine Handlungsvarietät ist: Je größer die Varietät eines Systems ist, desto mehr kann es die Varietät seiner Umwelt durch Steuerung vermindern. Daraus folgt, dass die Varietät des Steuerungssystems mindestens ebenso groß sein muss wie die Varietät der auftre- 
tenden Störungen, damit es die Steuerung ausführen kann. Folgt man dieser Idee, so bedeutet das: Wann immer es darum geht, erfolgreich mit hoch komplexen und dynamischen Situationen umzugehen, muss das handelnde System mindestens die gleiche Komplexität und Dynamik aufweisen wie das System, in dem gehandelt wird. Überträgt man diesen Gedanken auf heute, bedeutet dies: Wenn der Markt sich immer weiter vernetzt, wird es immer wichtiger, freie Vernetzung auch im eigenen Unternehmen zuzulassen und zu fördern. Ansonsten würde man Gefahr laufen, abgehängt zu werden.

Wie können Unternehmen nun konkret auf komplexe Systemanforderungen reagieren? Peter Kruse, Professor und Gründer der Unternehmensberatung nextpractice, weist darauf hin, dass Hierarchie zwar geordnetes und kalkulierbares Vorgehen sichert, aber keine empfehlenswerte Antwort auf die komplexe Dynamik von Netzwerken sei (Kruse 2015). Führung habe dann nicht mehr die Aufgabe, vorzudenken oder die Aktivitäten von Mitarbeiterinnen und Mitarbeitern zu steuern, sondern dafür zu sorgen, dass die Menschen im Unternehmen die notwendigen Zukunftskompetenzen entwickeln können, um Zusammenhänge zu erkennen und sich so selbst den Marktanforderungen entsprechend organisieren zu können. Netzwerkbildung ist damit beides, das Problem und die Lösung.

Die Anpassung an eine neue Organisationsrealität durch Lernen wird zu einem Schlüsselelement. Eine Studie von Peter Kruse (2009) zeigt: 77 Prozent der befragten Führungskräfte sind überzeugt, dass es einen grundlegenden Wandel im System von Führung braucht. Weitgehender Konsens herrscht unter den befragten Führungskräften darüber, dass es immer wichtiger wird, sich auf ergebnisoffene Prozesse einzulassen. Einheitlich wird mehr Mut zu iterativ-testender Agilität gefordert (Kruse 2015). Somit tritt an die Stelle des traditionellen Managens zwischen Soll und Ist die Aufforderung, sich schrittweise ausprobierend und lernend vorwärtszubewegen. Ziele werden nicht vorgegeben oder ausgehandelt, sondern gemeinsam immer wieder neu angepasst und entwickelt. Anhand von Kruses Studien kann das dazugehörige Idealbild von Führung auf drei zentrale Forderungen heruntergebrochen werden: Netzwerkorganisation statt Linienhierarchie, Selbstorganisation statt Steuerung und Kooperation statt Wettbewerb (ebenda).

Insgesamt ist zu beobachten, dass dem einzelnen Individuum eine zunehmend stärkere Verantwortung in der Organisation zukommt. Dass weniger Verantwortung an zentrale Führungsstrukturen abgegeben werden kann. Und dass drittens, die Frage, welche Future Skills eigentlich wichtig sind und gebraucht werden, um die jeweiligen Aufgaben in der „Netzwerkorganisation“ erfolgreich bearbeiten zu können, nur sehr personalisiert und im jeweiligen Kontext beantwortet werden kann - und das Erlernen dieser eben auch durch das Individuum geschehen muss. 
Geht man gegenwärtig in heutige Institutionen, so zeigt sich dieser Zusammenhang sofort. Den Verantwortlichen, die für die Future Skills Studie befragt wurden, ist klar, dass die Entwicklung der notwendigen Fähigkeiten so volatil und stetig im Wandel begriffen ist, dass 80 Prozent des notwendigen Lernens „on-the-job“ stattfindet. Dabei spielt das extern organisierte, formale und explizite Training eine immer unwichtigere Rolle. Die Reflexion, was Mitarbeiterinnen und Mitarbeiter eigentlich brauchen, und wo sie die notwendigen Kompetenzen erlernen können, passiert laut Einschätzung zu 90 Prozent aus Eigeninitiative. Die allgemeine Einschätzung ist die, dass es eine zunehmend untergeordnete Rolle spielt, kodifiziertes Wissen abrufbar parat zu haben. Viel wichtiger ist es, Daten, Information und Wissen zu suchen und zu diskriminieren, Unterscheidungen treffen zu können, sowie die Validität und Objektivität der jeweils gefundenen Informationen vornehmen zu können. Beispielsweise auch mit Suchmaschinen wie Google und Co. Die Fähigkeit, des eigenen individuellen Informationsmanagements ist dabei nicht nur eine Frage des subjektiven Wissensmanagements, also, wie man etwa seinen eigenen Wissensbereich organisiert. Sondern es geht auch darum, die Fähigkeit zu haben, Daten und Informationen zu validieren. Die im Netz vielfach verbreitete Ansicht, dass Informationen sich durch die Wiedergabe Vieler selbst validieren, ist dabei nicht immer zutreffend, ganz entgegen dem populären Verständnis des Wisdom of the Crowds.

Eine wichtige Zusatzbedingung hat schon im 18. Jahrhundert der französische Philosoph und Mathematiker Marquis de Condorcet aufgezeigt. Das Condorcet-Jury-Theorem besagt: Wenn die Menge des verteilten Wissens in den Köpfen von der an einer Schätzaufgabe beteiligten Menge von Entscheidern unter Zufall liegt, dann ist die Trefferquote der Gesamtentscheidung extrem gering. ${ }^{2}$ Liegt das Wissen der Einzelnen dagegen auch nur ein wenig über dem Zufall, dann schaukelt sich die Gruppe zu einer überraschend hohen Treffsicherheit auf. Peter Kruse, Zukunftsforscher, beschrieb diesen Zusammenhang einmal so:

„Wenn Sie bei Günther Jauch sitzen und eine Frage zu den Urlaubsvorlieben eines Prominenten gestellt bekommen, dann können Sie, weil „Promis“ über die Medien zu öffentlichen Personen werden, davon ausgehen, dass das Wissen der einzelnen Studiogäste über Zufall liegt. Hier sollten Sie den Publikumsjoker ziehen. Haben Sie aber eine Frage im Bereich Nuklearphysik, dann ist die Wahrscheinlichkeit recht gering, dass das Wissen über Zufall liegt. Dann können Sie besser gleich würfeln.“

2 Auch als Sozialwahltheorie bekannt, die Mitte des 20. Jahrhundert von Kenneth Arrow erfunden wurde (Arrow 1963). 
Bezogen auf Organisationen heißt das, dass das verteilte Wissen durch Informationsmonopole, Beziehungsnetzwerke oder hierarchische Schwellen prinzipiell limitiert wird. Eine wichtige Aufgabe ist es also dafür zu sorgen, dass eben dies nicht geschieht und Wissen frei verfügbar und ohne die typischen Wissensbeschränkungen (beispielsweise Informationsmonopole) in der Organisation verfügbar ist. Darüber hinaus gilt es genau zu überlegen, wie man mit der Validität der Informationen im Internet umgeht, seien sie auch durch noch so viele andere zitiert und vervielfältigt (Kruse in Personalwirtschaft 2015).

\section{II.1 Die Relativität des Fachwissens}

In Bezug auf Fachkompetenz und Wissensbestände zeigt die Future Skills Studie, dass sich in vielen Organisationen immer mehr die Erkenntnis durchsetzt, dass die Fähigkeit zur Initiative, und noch pointierter formuliert, zur Selbstinitiative, also dem Nachgehen und Umsetzen der individuell aus sich heraus entspringenden Impulse und Ideen und die damit eng verbundene Selbstkompetenz eine gleichwertige, wenn nicht vielleicht sogar wichtigere Rolle spielt als das Fachwissen. In dieser Polarisierung von Wissen einerseits und Kompetenz andererseits liegt jedoch nur ein scheinbarer Widerspruch. Denn Wissen ist kein von Kompetenz unabhängiger, sondern ein geradezu zentraler Baustein für Kompetenz. Kompetenz geht jedoch weit über Wissen hinaus (siehe dazu auch Kapitel B 1.2.2 Kompetenz). So bezeichnet Selbstkompetenz beispielsweise die

\footnotetext{
„Bereitschaft und Fähigkeit, als individuelle Persönlichkeit die Entwicklungschancen, Anforderungen und Einschränkungen in Familie, Beruf und öffentlichem Leben zu klären, zu durchdenken und zu beurteilen, eigene Begabungen zu entfalten sowie Lebenspläne zu fassen und fortzuentwickeln. Sie umfasst Eigenschaften wie Selbstständigkeit, Kritikfähigkeit, Selbstvertrauen, Zuverlässigkeit, Verantwortungs- und Pflichtbewusstsein. Zu ihr gehören insbesondere auch die Entwicklung durchdachter Wertvorstellungen und die selbstbestimmte Bindung an Werte." (KMK 2011)
}

Diese Erkenntnis ist oft Thema eines scheinbaren Widerspruchs, der in der jüngsten Debatte um Wissen/ Fachkompetenz vs. Handlungskompetenz und Fähigkeiten immer wieder auftaucht und einer grundlegenden Reflexion bedarf. Er drückt sich in Aussagen großer Tech-Companies (Times Higher Education 2015) über die Relativierung von formalen Abschlusszeugnissen genauso aus, wie in kontroversen Debatten von Lehrenden über die Frage, ob Kompetenzen überhaupt ein realistisches Ziel für Lernprozesse seien, wo es doch zunächst so viel Wissen zu erlernen 
gäbe. Insgesamt herrscht oftmals eine falsche Vorstellung über den Zusammenhang von Kompetenz und Wissen. Wir haben daher diesem Thema ein eigenes Kapitel gewidmet, um zu zeigen, dass wir es heute tatsächlich mit einer Kompetenzwende zu tun haben (siehe dazu auch Kapitel B 1.2.3 Kompetenz).

In den befragten Organisationen wurden die Personalentwicklungsinstrumente zunehmend darauf ausgerichtet, individuelle Kompetenzentwicklung und insbesondere die Entwicklung von Subjektkompetenzen (siehe Kapitel A2 Das Future Skills Triple Helix-Modell der Handlungsfähigkeit in emergenten Praxiskontexten) zu unterstützen. Dabei kommen vor allem coachingorientierte Methoden zum Einsatz. Dies stellt wiederum eine erhöhte Anforderung an Führungskräfte und macht in vielen Fällen ein Überdenken der bestehenden Governancestrukturen der Organisationen notwendig.

Die Interviews zeigen, dass es immer stärker weg vom Vermitteln hin zum selbstorganisierten Lernen geht. Die Entwicklung des selbstorganisierten Lernens als die Zukunftskompetenz schlechthin erfordert wiederum neue und besondere Modelle der Ausbildung, Unterstützung und Entwicklung bei Mitarbeiterinnen und Mitarbeitern. Diese sind für die Personalentwicklung in Organisationen auf allen Ebenen, sowohl bei der Programmgestaltung, also auch bei der individuellen Betreuung von Mitarbeiterinnen und Mitarbeitern, als auch auf didaktischer Ebene einer einzelnen Fortbildungsveranstaltung von hoher Bedeutung.

In fast allen befragten Organisationen gibt es Instrumente und Methoden zur Kompetenzerfassung, sowohl zu Beginn von Maßnahmen als auch später, die darauf abzielen, Mitarbeiterinnen und Mitarbeiter zu unterstützen, die eigene Entwicklung zu reflektieren. Führungskräfte verstehen sich zunehmend als Coaches, Lernbegleiterinnen und Lernbegleiter, weniger als diejenigen die Arbeitsvorgänge vorstrukturieren. Persönliche Dominanz und starke Persönlichkeit sind demnach out. Effiziente Zielerreichung und das Steuern über Kennzahlen werden als unzureichend erachtet. Vor dem Hintergrund wachsender Dynamik und Komplexität bewerten die Führungskräfte die Erfolgskonzepte von gestern intuitiv als Risiko von morgen.

\section{II.2 Der Future Skills Turn}

Es ist eine regelrechte Wende in Richtung Future Skills zu beobachten. Diese drückt sich auch in den Instrumenten aus, die in der Personalentwicklung mehr und mehr eingesetzt werden. So berichtet ein mittelständisches Unternehmen im Bereich Medizinprodukte, dass es Feedbackbögen für die Mitarbeiterinnen und Mitarbeiter 
einsetzt, denen neun Kompetenzen zugrunde liegen, von denen lediglich noch eine tatsächlich fachlich ist. In der Personalentwicklung wird immer mehr Wert daraufgelegt, wie Zusammenarbeit und Vernetzung gefördert werden können. So werden beispielsweise Persönlichkeitsmodelle und -tests eingesetzt, die dabei helfen $\mathrm{zu}$ verstehen, welche Präferenzen bei der Kooperation der Mitarbeiterinnen und Mitarbeitern vorliegen und wie das gegenseitige Verständnis und die Bereitschaft für Kooperation verbessert werden können (Mittelständische Bank).

Der neue Fokus auf Future Skills zeigt sich auch mit Blick auf Weiterbildungsangebote und -maßnahmen. Fortbildungsangebote sind weniger katalogorientiert, sondern zielen verstärkt auf Netzwerkbildung - und damit auf Selbstorganisation (siehe dazu auch Kapitel B 2.1 „Drift to Self-Organisation“: Selbstorganisation als gesellschaftliches Leitprinzip). Ganz praktisch drückt sich dies beispielsweise darin aus, dass eine Personalverantwortliche berichtet, es gäbe heute ca. 200 Angebote der Personalentwicklung pro Jahr, und 80-85 Prozent davon seien als Kollege schult Kollege organisiert (Mittelständischer Medizinproduktehersteller). In manchen Organisationen gibt es zudem explizit Abteilungen, die den Stellenwert des Lernens für die Arbeit herausstellen und beide Themen miteinander koordinieren, beispielsweise ein Team Lernen und Arbeit in einer der teilnehmenden Organisationen (große Drogeriemarktkette).

Die Future Skills-Wende - weg vom Fachwissen hin zu Future Skills - zeigt sich auch darin, dass neben den traditionellen Personalentwicklungsinstrumenten nun Coaching, Beratung und Mentoring eine immer größere Rolle spielen. Dabei steht Coaching für ergebnisoffene und lösungsfokussierte Unterstützung der persönlichen Kontexte, Beratung für ein Format, in dem es vor allem um gezielte Unterstützung für ein vorliegendes Problem geht, während Mentoring auch zwischen Kolleginnen und Kollegen unterschiedlicher Expertise stattfinden kann. Zukunftsweisend dabei ist die Auflösung von Grenzen des Privaten und des Professionellen. In einer der befragten Organisationen können Mitarbeiterinnen und Mitarbeiter auch Themen aus ihrem privaten Umfeld mit in ein Coaching einbringen. Das macht insofern Sinn, als dass gerade beim Coaching als ergebnisoffenem Format immer auch Fragestellungen aus dem privaten, persönlichen eine Rolle spielen und der professionelle Kontext nicht immer trennscharf dagegen abgegrenzt werden kann. Voraussetzung ist dabei das Schaffen eines Kontextes, in dem klare Informationsschranken definiert sind und eine Vertrauenskonstellation aufgebaut wird.

Eine der Organisationen hat, auf dem Mentoring-Format aufbauend, einen zusätzlichen Ansatz eingeführt: das Reverse Mentoring. Es wird dabei kein Mentoringangebot definiert, sondern ein Mentoringbedarf formuliert, der dann von Kollegen - vor allem auch aus anderen Abteilungen oder hierarchischen Kontexten bedient werden kann. Eine Auszubildende oder ein Auszubildender schult so vielleicht das 
Vorstandsmitglied in einem bestimmten Softwarethema oder Mitarbeiterinnen und Mitarbeiter aus dem Vertrieb sind Mentoren für die Expertinnen und Experten aus der Abteilung Entwicklung (mittelständische Bank).

\section{II.3 Förderung von Future Skills: Einblicke in die Praxis}

Wie Organisationsstruktur, Werte, Führungs- und Kommunikationsstrukturen zusammenspielen müssen, um eine Organisationskultur für Future Skills aufzubauen, zeigen folgende Beispiele.

\section{II.3.1 Kompetenzwerkstatt: Vernetzung und Selbstorganisation stärken}

Mitarbeiterinnen und Mitarbeiter aller Abteilungen und Hierarchiestufen können sich in eine einjährige Kompetenzwerkstatt einschreiben. Ziel der Kompetenzwerkstatt ist es, eine persönliche Lern- oder Entwicklungsaufgabe anzugehen. Dazu können Schulungen, Trainings oder kollegiale Beratung/ Schulung in Anspruch genommen werden. Wichtig: Es können sowohl Themen, die für den professionellen Arbeitszusammenhang von Bedeutung sind, als auch Themen, die aus privatem Interesse relevant erscheinen, gewählt werden. Zentrales Element der Kompetenzwerkstatt ist die regelmäßig stattfindende Reflexions- und Sharingwerkstatt. Hier geht es darum, dass alle Teilnehmenden sich untereinander davon berichten, was sie bislang gelernt haben, wie es vonstattenging und was als nächstes ansteht. Schwieriges, Fortschritte, Überraschendes und Unerwartetes stehen im Mittelpunkt der Kompetenzwerkstatt. Die Veranstaltungen finden außerhalb des eigentlichen Tagesgeschäftes statt und werden moderiert. Die Teilnehmenden reflektieren ihren Lern- und Entwicklungsfortschritt regelmäßig, entwickeln eine Sprache für das eigene Lernen.

Außerdem dient dieses Format der Vernetzung von Mitarbeitenden innerhalb der Organisation. Es entsteht ein neues Netzwerk von Mitarbeiterinnen und Mitarbeitern, das sich durch die Gesamtorganisation zieht und Mitarbeiterinnen und Mitarbeiter zusammenbringt, die vorher noch keinen Kontakt und keine Verbindung zueinander hatten. Die oberste Prämisse hierbei lautet: Es darf explizit sowohl um Privates als auch um Professionelles gehen. Auf diese Weise wird die Voraussetzung für eine stärkere Selbstorganisation geschaffen und verbessert. Den Abschluss einer jeden Kompetenzwerkstatt bildet eine eintägige Veranstaltung, bei 
der die jeweils persönlichen Lernreisen erzählt und miteinander geteilt werden. Auch der Vorstand der Organisation nimmt an diesem besonderen Ereignis teil.

\section{II.3.2 Vom Ende des Belehrens: Lernlinge und Studierende als Expertinnen und Experten}

Bei einer großen Drogeriemarktkette werden Lehrlinge und (duale) Studierende nicht als Lehrlinge oder Studierende bezeichnet, sondern mit einer neuen kreativen Wortschöpfung: „Lernlinge“. Dahinter steckt die Auffassung, dass Lernen nicht über Belehren oder Lehren funktioniert, sondern ein eigenaktiver und selbstgesteuerter Prozess ist. Alle darauf bezogenen Funktionen sind im Konzept entsprechend angepasst: Ausbilderinnen und Ausbilder sind Lernbegleiterinnen und Lernbegleiter, Zertifikate oder Prüfungen werden in einem persönlichen Lernpass dokumentiert, Lernbegleiterinnen und Lernbegleiter bekommen eine spezielle Ausbildung zur/zum Lernbegleiterin/Lernbegleiter, die insbesondere auch Lernen als selbstorganisierten Prozess unterstützen soll. Es gibt Lernveranstaltungen und Lernwerkstätten anstelle von Lehrveranstaltungen. Das Format der Lernwerkstätten zielt insbesondere auf die Unterstützung der Fähigkeit zum selbstorganisierten Lernen (siehe dazu auch Kapitel B 2.1 „Drift to Self-Organisation“: Selbstorganisation als gesellschaftliches Leitprinzip) ab, Thematisch-Inhaltliches ist dabei zunächst zweitrangig.

Bei einem Weltmarktführer im IT-Servicebereich wird die Blickrichtung umgedreht - nicht mehr Auszubildende und (duale) Studierende sind es, die ausgebildet und weitergebildet werden müssen, sondern das Unternehmen möchte von den Sichtweisen der jungen Menschen und deren unverstelltem Blick profitieren. So können sich einzelne Abteilungen mit Projektideen bei den Studierenden bewerben. Das Studium, die Weiterbildung und die Ausbildung vollziehen sich also nicht wie ein von vornherein durch das Curriculum festgelegter Prozess, zu dessen Beginn bereits feststeht, welche Inhalte in welcher Konstellation zu welchem Zeitpunkt gelernt werden können, sondern sind ein situativ zusammengestelltes Portfolio an Erfahrungen. Diese werden reflektiert, dokumentiert und auch durch Coaches und Mentoren betreut. Zentrales Merkmal hierbei ist, dass die Projekte, in denen Studierende eingesetzt werden, authentische Problemkontexte darstellen, also tatsächlich reale Probleme, die für das Unternehmen relevant sind. In besonderen Fällen geht dies soweit, dass Studierende als Intrapreneurs in Teams eigene Geschäftsideen entwickeln, die sie dann für das Unternehmen weiterentwickeln und die, bis zur Geschäftsreife getragen, dann eigene neue oder weiterentwickelte Produkte des Unternehmens bilden können. Auch in die Entwicklung von Zukunftsthemen sind Studierende eingebunden, indem sie aus ihrer Perspektive Analysen und Business 
Cases entwickeln, wie das Unternehmen mit neuen Themen am Markt umgehen kann. Ein Beispiel hierfür ist eine studentische Projektgruppe, die über das Thema Blockchain als Geschäftsfeld nachdenkt.

\section{II.3.3 Kreativität in verteilten Teams}

In den Interviews mit einem global führenden Technikkonzern weisen die Expertinnen und Experten darauf hin, dass es wichtig sei, als Organisation Kompetenz dafür aufzubauen, wie Kompetenzen als „shared expertise“ in Abteilungs- und Projektteams - auch teilweise weltweit - zusammengestellt werden können. Dabei liegt der Ansatz zugrunde, dass die zukünftig wichtigen Future Skills nicht alle als Fähigkeit bei Mitarbeiterinnen und Mitarbeiter vorliegen können, sondern dass es aus Sicht der Organisation gilt, möglichst ein vollständiges Kompetenzspektrum innerhalb eines Teams verteilt zu haben. Nicht nur Fähigkeiten zur Umsetzung von Vorhaben oder Expertise zum Bewältigen von definierten Herausforderungen sind dabei im Fokus, sondern gerade auch die Zusammenstellung von Mitarbeiterprofilen, die zu einem maximalen Grad an shared creativity als gemeinsamer Kreativität innerhalb des Teams führen. In vielen Fällen handelt es sich um weltweit verteilte Teams, die nur kurze Zeit zusammenarbeiten. Aus Sicht der Organisation geht es dabei darum, dass die vorhandenen Kompetenzen und Erfahrungen bekannt und dokumentiert sein müssen. Ein solches Talentmanagement aufzubauen ist eine umfassende Aufgabe des Human Capital Managements (Ehlers et al. 2003) und nur für Organisationen möglich, die einen besonders hohen Reifegrad an Personalmanagement implementiert haben. Dazu gehört auch ein hoher Grad der Formalisierung von Kompetenzen und Kompetenzanforderungen. Zusätzlich ist das Unterstützen von Kreativität eben genau in dem Spannungsfeld zwischen Formalisierung und Informalität angesiedelt, den es als Organisation explizit aufzubauen gilt - und das eher durch Regeln und Kategorien von HCM Systemen überstrukturiert und dadurch behindert wird.

\section{II.3.4 Flexibilisierung und Selbstorganisation}

Lernen und Arbeiten findet in allen befragten Organisationen in Kontexten statt, die eine Flexibilisierung von Arbeitsabläufen, Rollen sowie Funktionsbeschreibungen und -definitionen zulassen. Beispiele dazu sind etwa die Arbeitszeitorganisation am Shopfloor oder das Abschaffen von Arbeitszeitregelungen (bei einem großen 
Chemiekonzern). Bei einer teilnehmenden Organisation der Future Skills Studie wurde das Prinzip der Selbstorganisation bei Arbeitszeitregelungen in allen Filialen deutschlandweit eingeführt. Mitarbeiterinnen und Mitarbeiter können ihre Dienstpläne in Absprache untereinander ohne Genehmigungsverfahren von Vorgesetzten gestalten. Was hier so einfach klingt, ist bei sehr heterogenen Kontexten und Mitarbeiterinnen und Mitarbeitern eine enorme Herausforderung, die eben genau solche Future Skills erfordert, welche als Schlüssel für die zukünftige Arbeitswelt angesehen werden, nämlich Selbstorganisation und Metakompetenzen.

\section{II.3.5 Freiräume schaffen, Perspektiven wechseln, Innovation und Kreativität ermöglichen}

Wie bekommt man die Mitglieder einer Organisation dazu, über ihre jeweilige Situation hinaus zu denken und Vorschläge für neue Produkte, neue Geschäftsideen oder Abläufe zu entwickeln? Sogar dazu, die Intelligenz, Erfahrung und Perspektive aller Mitglieder der Organisation zu nutzen, um die Position des Unternehmens/ der Organisation einmal zu reflektieren und sich Ansatzpunkte für eine Positionierung in zehn Jahren zu überlegen?

Der Fall eines Weltmarktführers im Medizinproduktebereich zeigt, wie dies gehen könnte. Die Organisation hat hierzu einen unternehmensinternen Wettbewerb initiiert. Alle Unternehmensangehörigen waren aufgerufen, Vorschläge bei der Geschäftsführung einzureichen, wie eine neue Unternehmensstrategie aussehen könnte; Produkte, Marktplatzierung, zukünftige Stärken, USPs für die kommende Dekade waren gefragt. Das Besondere: Jede Einreichung konnte zudem in einem mündlichen Kurztermin erläutert werden. Alle wurden gehört. Aus allen Vorschlägen wurden einige ausgewählt, die besonders weitreichend und divers waren. Diejenigen, die sie eingebracht hatten, wurden dann als Team zusammen in ein siebenwöchiges Retreat geschickt. Sie bekamen die Aufgabe, in sieben Wochen in einer eigenen Umgebung, für die die Geschäftsleitung extra Büros angemietet hatte, die beste Zukunftsstrategie zu entwickeln, die ihnen möglich war. Der Clou war dabei, dass sie alle zusammen an dieser Zukunftsaufgabe arbeiten sollten. Menschen aus ganz unterschiedlichen Unternehmensbereichen, mit ganz unterschiedlichen Perspektiven und Ideen, die alle zusammen an diesem Großprojekt mitarbeiten konnten. Die neueste Forschung zum Thema Innovation und Kreativität zeigt eines eindeutig: Das Loslösen aus den Arbeitskontexten und Zusammenkommen in neuen sozialen Konstellationen fördert das divergente Denken und trägt somit zur Kreativität bei (Bezmen et al. 2015). 


\section{II.3.6 Selbstorganisation und selbstverantwortetes Lernen stärken}

Ein großer Chemiekonzern hat einen ganz eigenen Ansatz entwickelt, um Lernkompetenzen zu stärken. Traditionell bekamen neue Mitarbeiterinnen und Mitarbeiter sowie Führungskräfte in der Organisation unterschiedliche Trainings angeboten, die sie teilweise verpflichtend, teilweise auch freiwillig absolvieren konnten. Die Frage war dann, wie kommt man aus der Angebots- in eine Nachfragesituation? Wie kommt man aus der Rezeptionsmentalität in einen selbstverantworteten Lernprozess? Die Organisation erkannte, dass es nicht mehr möglich war, sich vollumfänglich um die Weiterbildung und die Lernbedürfnisse ihrer Mitarbeiterinnen und Mitarbeiter zu kümmern, da Lernen zum Schlüssel zukünftiger Organisationsgestaltung geworden ist. $\mathrm{Zu}$ individuell und divers waren und sind die Kontexte geworden, in denen Mitarbeiterinnen und Mitarbeiter lernen wollen, sollen und können. Daher wurde ein neues Konzept entwickelt. Zusammen mit einer pädagogischen Hochschule besprach das Unternehmen Konzepte für selbstorganisiertes Lernen und überlegte, wie diese in der Organisation eingebracht und gestärkt werden könnten. Ein radikaler Wandel, weg vom strukturierten Präsenzangebot in der Weiterbildung hin zum selbstverantworteten Lernen über E-Learning in virtuellen Welten wurde eingeleitet. Nicht mehr definierte Anforderungen, sondern reiche Lernwelten wurden den Mitarbeiterinnen und Mitarbeitern angeboten. Zunächst wurden zwölf Module entwickelt, die den Mitarbeiterinnen und Mitarbeitern zur Verfügung gestellt wurden. Aus ihnen konnten sie ihren Bedürfnissen entsprechend auswählen, was für sie richtig und passend - gemessen an ihrem jeweiligen Kontext - war. Immer mehr Module wurden so entwickelt und eine reichhaltige Lernwelt entstand, die nun den unterschiedlichen Zielgruppen zur Verfügung steht. Die Zukunft liegt darin, dass nicht mehr zentral bestimmt und gesteuert wird, wer wann was lernt, sondern darin, dass Mitarbeiterinnen und Mitarbeiter zunehmend selbst zu den Managerinnen, Managern und Gestaltenden ihrer eigenen Lernerfahrungen werden. Nur sie wissen, was sie zur Stärkung ihrer jeweiligen Professionalität und welches Wissen und welche Kompetenzen sie für die Entwicklung ihres eigenen Geschäftsbereiches benötigen. Führungskräfte sind dann in einer neuen Rolle. Freiräume schaffen und Strukturen für Selbstverantwortung und Selbstorganisation ermöglichen. Führungskräfte, so die Erfahrung, müssen Mut machen, Mitarbeiterinnen und Mitarbeiter auf ihrem Weg zu mehr Selbstverantwortung coachen und Arbeitssituationen flexibel gestalten, sodass Lernen ermöglicht wird. 


\section{II.3.7 Persönlichkeiten und Selbstbewusstsein stärken}

Future Skills können nicht (nur) kognitiv erlernt werden, sondern haben mit der Entwicklung der Persönlichkeiten und Stärkung der Professionalität zu tun. Dass die Zukunft der Kompetenzorientierung in Organisationen relevant ist, zeigt auch das Beispiel von Personal- und Organisationsentwicklungsangeboten, in denen es um die Stärkung der eigenen Persönlichkeit geht. Doch wie geht das vonstatten? Wie kann man Mitarbeiterinnen und Mitarbeiter in ihrem eigenen Selbstbewusstsein stärken? Es sind tatsächlich gezielte Coachingmaßnahmen erforderlich, die zu mehr Selbstorganisationsfähigkeit, Autonomie und Handlungsfähigkeit führen, gerade für Kontexte in denen Mitarbeiterinnen und Mitarbeiter immer mehr mit Unsicherheit und Ambiguität umgehen müssen. In diesen Kontexten müssen sie Entscheidungen treffen und Verantwortung in Situationen unvollständiger Informationsverfügbarkeit übernehmen. Oftmals ein schwieriges Unterfangen. Wie stärkt man nun ihr Selbstbewusstsein dabei?

Eine an der NextSkills Studie teilnehmende Organisation führt zu diesem Zweck mit Mitarbeiterinnen und Mitarbeitern beispielsweise Theaterworkshops durch. Die Teilnehmenden, die zum ersten Mal auf der Bühne stehen und dort ihren Part bewältigen, machen persönliche Erfahrungen, in denen sie sich nicht nur selbst auf ganz neue und andere Weise erleben, sondern auch ihre Kolleginnen und Kollegen. Geht es im Alltag vielleicht gerade darum, das, was als Schwäche an der eigenen Person wahrgenommen wird, eher vor den Kolleginnen und Kollegen zu verbergen, so geht es auf der Bühne genau darum, sich zu zeigen, auch und gerade in allem Unvermögen. Alle sitzen dabei zunächst im gleichen Boot. Für vermutlich die meisten ist die Bühnenerfahrung im Theaterworkshop eine neue, eine Referenzerfahrung. Als Trainerin, Trainer und Coach geht es darum, deutlich zu machen, dass nicht Brillanz oder absolutes Können zählen, sondern darum, Lernen und Entwicklung zu ermöglichen, sich auf die Situation einzulassen. Ist das verinnerlicht, können Mitarbeiterinnen und Mitarbeiter - so die Idee - miteinander zukünftig alle neuen Herausforderungen ebenso gemeinsam in Teams und in Transparenz ihrer Stärken und Schwächen bewältigen.

Auch andere Organisationen setzen auf Gruppenerfahrung und Gruppendynamik. Dabei geht es oft darum, genau diejenigen in Organisationen zusammenzubringen, die sonst nichts miteinander zu tun haben, also abteilungs- oder geschäftsbereichsübergreifend zu agieren und das oft in ganz neuen und externen Umgebungen. Das reicht von Wochenenden im Kloster bis hin zu einem Besuch im Kletterpark. Future Skills, deren Entwicklung in diesen Trainings im Vordergrund steht, sind neben Selbstbewusstsein, Selbstkompetenz und einer Stärkung des Selbstwerts auch Autonomie und Leistungsmotivation. 


\section{II.3.8 Freiräume schaffen}

Entwicklung braucht Freiräume, braucht Anerkennung und das Wissen darum, dass das eigene Engagement auch ankommt, und dass Gestaltungsvorschläge umgesetzt werden können. Eine der an der NextSkills teilnehmenden Organisationen lebt dies als praktische Realität. Das betriebliche Vorschlagswesen wird hier ernst genommen. Die Mitarbeiterinnen und Mitarbeiter können online ihre Ideen einbringen und dann über partizipative Auswahlprozesse schrittweise zu einer Realisierung kommen. Dabei werden beispielsweise Vorschläge dazu eingebracht, wie das Werksgelände der Zukunft aussehen kann. Es geht dann darum, ob das Gelände auch für einen Teil der Öffentlichkeit geöffnet werden kann, um der Bevölkerung näherzubringen, was dort erforscht und produziert wird, oder um einfach mehr in die Community, das Stadtviertel, das Stadtbild integriert zu sein. Die Identität der einzelnen Mitarbeiterinnen und Mitarbeiter mit ihrem Tun, ihrer Organisation kann so noch gestärkt werden. Kann beispielsweise eine Gaststätte auf dem Werksgelände eröffnet werden? Ist es sinnvoll, eine Wäscherei für die Werksmitarbeiterinnen und -mitarbeiter direkt auf dem Werksgelände zu eröffnen? Die Change Agents, deren größere und kleinere Vorschläge zur Umsetzung ausgewählt werden, bekommen Freistellungen dafür, diese in die Tat umzusetzen. Das Beispiel zeigt, dass Future Skills nicht für sich alleinstehen, um wirksam zu werden, sondern idealerweise durch eine offene, mitarbeiterorientierte Führungskonzeption unterstützt werden. Diese müssen ausprobiert und umgesetzt werden, um die Organisationsstrukturen, Abläufe und die gesamte Organisation so zu gestalten, dass die Zukunft der Arbeit mit hoher Identität, Freiräumen für kreatives Umdenken möglich werden.

\section{II.3.9 FUSE: Beteiligungsorientierte Strategieentwicklung}

Ein letztes Beispiel - diesmal aus der Hochschulwelt - zeigt, wie wirkliche partizipative Gestaltung von zukünftigen Organisationsstrategien aussehen kann, und warum diese wichtig sind. Die Dublin City Universität in Irland befand sich 2017 in einer tiefen Restrukturierung. In dieser Situation war der Präsident damit beauftragt, eine neue Fünf-Jahresstrategie zu entwickeln. Die Hochschulgremien pochten darauf, dass dies unter maximalem Einbezug aller Beteiligten geschehen sollte, um die Akzeptanz und Durchsetzungsstärke zu erhöhen - und nicht Gefahr zu laufen, einen bürokratischen Papiertiger zu erschaffen. Es wurden zehn Strategiegruppen eingesetzt, die zu zehn unterschiedlichen Themenstellungen Zukunftsentwürfe entwickelten. Das Kernstück der Strategieentwicklung aber trug den Namen „FUSE“. FUSE war eine Crowdsourcing Initiative der Dublin City University, in der es 
darum ging, Kernideen für die zukünftige Fünf-Jahresstrategie der Hochschule zu entwickeln - und zwar gemeinsam mit allen Stakeholdern der Hochschule. FUSE ist als Brainstorming Event konzipiert, in der alle 17.000 Studierenden, 80.000 Alumni und 1.200 Hochschulangehörigen die Möglichkeit hatten, ihre Ideen online beizutragen - 30 Stunden lang. Das FUSE Event wurde von Richard Bruton, TD, Minister for Education \& Skills zusammen mit dem Hochschulpräsidenten eröffnet. Es fanden Debattierstunden der Studierenden, der Mitarbeiterinnen und Mitarbeiter und der Lehrenden statt, Sleep-Ins in der Hochschulbibliothek und Liveschaltungen aller Hochschulcampus, online TV Zwischenfazits und online Zuschaltungen der Hochschulpartner, der lokalen Politik und der Unternehmen. Über 7.500 Posts konnten so gesammelt werden. Diese wurden geclustert, Doppellungen vereinheitlicht, paraphrasiert und zu Themenclustern zusammengeführt. Verschiedene Teams arbeiteten Kernaussagen heraus, sodass der Hochschulleitung ein reiches Bild an klaren Themenprioritäten für die zukünftige Strategie präsentiert werden konnte. Es ist ein Kennzeichen zukünftiger Organisationen, Beteiligung an der Gestaltung zuzulassen. Dabei geht es vor allem darum, die Identifikation der Organisationsmitglieder mit ihren Organisationen zu erhöhen - nicht nur in der Arbeitswelt, sondern eben auch in der Bildungs- und Schulwelt.

In Hochschulen dreht dies die Entwicklungsrichtung um. Sind bisher die Studierenden die Belehrten, die anhand von fest vorgegebenen Curricula zu fest vorgegebenen Zeiten lernen, so geht es zukünftig darum, sie zu befragen, und zu bitten, ihre Vorschläge für die Hochschulentwicklung einzubringen. Wo soll die Reise hingehen? Was sind die zukünftig wichtigen Themen, die Beachtung finden müssen? Welche Services werden benötigt? Und wie soll Studieren erfahrbar sein? Partizipation und Beteiligung der Organisationsmitglieder ermöglicht Gestaltung. Sie fördert aber auch die Selbstverantwortung - als Voraussetzung für Selbstorganisation - und nimmt Mitarbeiterinnen und Mitarbeiter in die Pflicht. Organisation wird nicht mehr als etwas extern Vorgegebenes erfahren, Strukturen, die gewissermaßen zwangsweise vorhanden sind und in die jeder seine eigene Kreativität und Vorstellung einfügen muss, sondern es ist tatsächlich die Struktur, die über Beteiligung beeinflusst werden kann. Die man dadurch auch selbst verantwortet, mitverantwortet.

Die Gestaltung von gemeinsam getragenen Verantwortungsstrukturen ist eine der größten Herausforderungen in der zukünftigen Arbeitswelt. Sie ist das wichtigste Bindeglied in der Kette: Beteiligung - Einfluss und Identifikation - Selbstverantwortung - Selbstorganisation. Sie hat Einfluss darauf, weil sich Organisationsmitglieder entwickeln wollen und müssen. Freiräume, Eigenverantwortung, die Fähigkeit zum Mitteilen, zur Beteiligung, all dies sind zugleich Kennzeichen und Ergebnisse der neuen Kultur von Arbeit und Lernen. 
Open Access Dieses Kapitel wird unter der Creative Commons Namensnennung 4.0 International Lizenz (http://creativecommons.org/licenses/by/4.0/deed.de) veröffentlicht, welche die Nutzung, Vervielfältigung, Bearbeitung, Verbreitung und Wiedergabe in jeglichem Medium und Format erlaubt, sofern Sie den/die ursprünglichen Autor(en) und die Quelle ordnungsgemäß nennen, einen Link zur Creative Commons Lizenz beifügen und angeben, ob Änderungen vorgenommen wurden.

Die in diesem Kapitel enthaltenen Bilder und sonstiges Drittmaterial unterliegen ebenfalls der genannten Creative Commons Lizenz, sofern sich aus der Abbildungslegende nichts anderes ergibt. Sofern das betreffende Material nicht unter der genannten Creative Commons Lizenz steht und die betreffende Handlung nicht nach gesetzlichen Vorschriften erlaubt ist, ist für die oben aufgeführten Weiterverwendungen des Materials die Einwilligung des jeweiligen Rechteinhabers einzuholen.

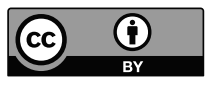

